\title{
Thoracic Epidural Catheterization Using Ultrasound in Obese Patients for Bariatric Surgery
}

\author{
Tomoki Nishiyama \\ Department of Anesthesiology, Shinagawa Shishokai Hospital, Tokyo, Japan \\ nishit-tky@umin.ac.jp
}

Received date: 23 November 2013; Accepted date: 16 January 2014; Published date: 7 March 2014

Academic Editor: Andréa Ramalho

Copyright @ 2014. Tomoki Nishiyama. Distributed under Creative Commons CC-BY 3.0

\begin{abstract}
Objective: We investigated the usefulness of ultrasound for a thoracic epidural catheterization in bariatric surgery. Methods: Sixteen patients with $125.9 \mathrm{~kg}$ in mean body weight and $45.5 \mathrm{~kg} / \mathrm{m}^{2}$ in mean body mass index were studied. The longitudinal view of the ultrasound scanning was used to detect the spinous process and ligamentum flavum-dura matter unit. The distance from the skin to the ligamentum flavum-dura matter unit was measured as an ultrasound depth and corrected by the angle of the epidural needle. An epidural puncture was performed by a median approach with a loss of resistance method. The distance from the skin to the tip of the needle was measured as a needle depth. Multiple regression analysis and Bland-Altman plot were used to judge the relation between the needle depth and the corrected ultrasound depth. Results: Epidural catheterization was successfully performed at the first attempt in 14 patients and second in two patients. The needle depth was 9.1 (range, 5.5-11.0) $\mathrm{cm}$ and the ultrasound depth was 6.5 (4.0-8.5) $\mathrm{cm}$. The correlation coefficient $\left(\mathrm{r}^{2}\right)$ of the corrected ultrasound depth and the needle depth was 0.81 . The Bland-Altman analysis showed the bias of $1.8 \mathrm{~cm}$ and limits of agreement of $0.63 \mathrm{~cm}$ and $2.94 \mathrm{~cm}$. Discussion: Ultrasound was useful to insert a thoracic epidural catheter in obese patients receiving bariatric surgery, while the measurement of the ultrasound depth could not be a surrogate measurement of the needle depth.
\end{abstract}

Keywords: bariatric surgery, epidural block, morbid obese, ultrasound

\section{Introduction}

An epidural catheterization is usually difficult in morbid (body mass index (BMI) > $35 \mathrm{~kg} / \mathrm{m}^{2}$ ) or super obese (BMI $>55 \mathrm{~kg} / \mathrm{m}^{2}$ ) (Adams \& Murphy, 2000) patients. Therefore, it is rarely used for anesthesia in bariatric surgery. However, an epidural catheterization is commonly used for anesthesia and postoperative analgesia in abdominal surgery. We should use an epidural block in bariatric surgery, too. We have already summarized the anesthesia of 20 bariatric surgeries performed between November 2008 and March 2010 (Nishiyama, et al, 2012). In

Cite this Article as: Tomoki Nishiyama (2014),"Thoracic Epidural Catheterization Using Ultrasound in Obese Patients for Bariatric Surgery", Journal of Research in Obesity, Vol. 2014 (2014), Article ID 538833, DOI: $10.5171 / 2014.538833$ 
that study, we inserted an epidural catheter in 16 patients. The present report focused on an epidural catheterization in these 16 patients and analyzed the data of epidural catheterization.

\section{Methods}

An epidural catheterization was performed on the day before surgery in 12 patients and just before anesthesia induction in 4 patients. The patients were put on the left lateral position on the operating table. At first, the midline and the location of the spinous process were checked manually. Then an ultrasound ( $\mathrm{M}$ turbo ${ }^{\mathrm{TM}}$, Sonosite Japan, Tokyo, Japan) with a curved array probe (2 - $5 \mathrm{MHz}$ ) was used. At first, the scanning was performed by placing the probe in transverse orientation to detect the midline and 12th rib. Then, the probe was placed in longitudinal orientation to detect the spinous process and ligamentum flavum-dura matter unit of the target spinal level (Figure 1). The distance from the skin to the ligamentum flavum-dura matter unit was measured as an ultrasound depth.
Then, $1 \%$ lidocaine was injected as a local anesthesia at the prospected epidural needle insertion point, which was the center of the two spinous processes shown by the ultrasound. An epidural puncture was performed using a $12 \mathrm{~cm} 17 \mathrm{G}$ epidural needle (Unisis, Saitama, Japan), with the angle between 60 and 90 degrees against the skin by a median approach. An epidural space was recognized by a loss of resistance method using air or saline, and a catheter was inserted $5 \mathrm{~cm}$ cephalad. Lidocaine $1 \% 3 \mathrm{~mL}$ was administered to check whether a catheter was not in the spinal space. When we could not recognize an epidural space by a loss of resistance method until the needle was inserted to the bone, the first attempt was judged as failed and we tried the second attempt. The distance from the skin to the tip of the needle was measured as a needle depth and the angle of the needle to the skin was measured. According to the angle of the needle to the skin, ultrasound depth was corrected to the corrected ultrasound depth by the formula of (corrected ultrasound depth $)=($ ultrasound depth) $/$ sin (angle) .

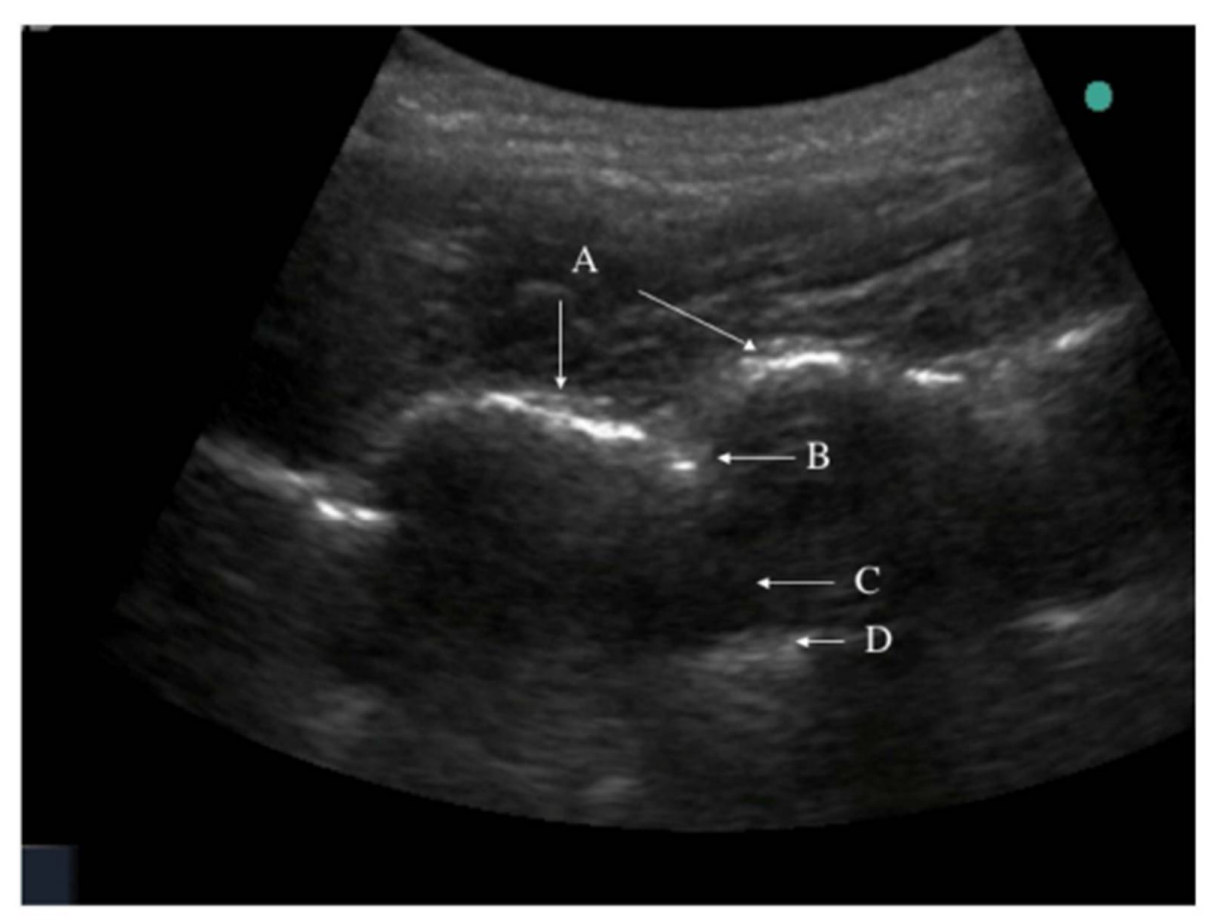

Figure 1: Ultrasound image of longitudinal view

A, spinous process; B, Ligamentum flavum- dura matter unit; C, dural sac; D, vertebral body; the distance between skin to $B$ was measured as the ultrasound depth. 
Multiple regression analyses were used to analyze the relation of the needle depth and other factors, such as the corrected ultrasound depth, height, body weight, ideal body weight, and BMI. Bland-Altman plot was also used to judge the relation between the needle depth and the corrected ultrasound depth.

\section{Results}

Patients were 6 males and 10 females, 39 (range, 21 - 59) years old, 166 (152 - 181) $\mathrm{cm}$ in height, 125.9 (90.8 - 179.0) kg in body weight, and 45.5 (35.8 - 64.4) kg/m² in BMI. Laparoscopic sleeve gastrectomy was done in 10 patients, laparoscopic gastric bypass in one patient, open gastric bypass in 3 patients, open sleeve gastrectomy in one patient, and open duodenal switch was in one patient.

The spinous process was not recognized manually in all patients. Ultrasound showed spinous process and the ligamentum flavum-dura matter unit in all patients (Figure 1); and all the epidural catheterization was successfully performed, in 14 patients at the first attempt and in two patients at the second attempt.

The time from the start of ultrasound scan to the end of epidural catheterization was 9.5 (range, 6.0 - 12.5) minutes. An epidural catheterization was performed at Th6-7 in 13 patients, Th7-8 in one patient, Th8-9 in one patient, and Th9-10 in one patient. The needle depth was 9.1 (range, $5.5-11.0) \mathrm{cm}$, and the ultrasound depth was $6.5(4.0-8.5) \mathrm{cm}$. The angle of the needle to the skin was $64(60-75)$ degrees.

When the ultrasound depth was corrected by the angle, $\mathrm{Y}$ (needle depth) $=1.18+$ $0.93 \mathrm{X}$ (corrected ultrasound depth) $\left(\mathrm{r}^{2}=\right.$ 0.81) (Figure 2). The corrected ultrasound depth was 1.6 (range, $0.8-2.5$ ) $\mathrm{cm}$ shorter than the needle depth.

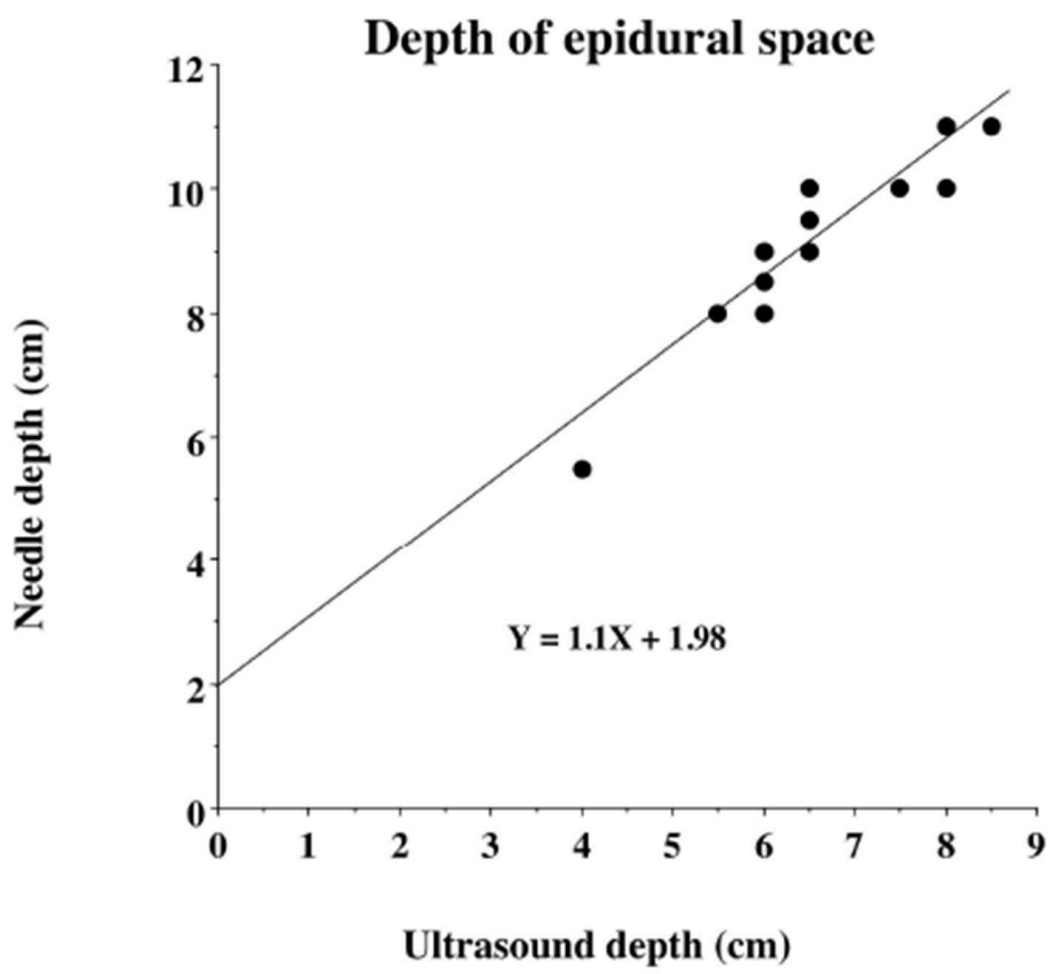

Figure 2: Relation of corrected ultrasound depth and needle depth The ultrasound depth was corrected by the angla of the needle to the skin. $\mathbf{r}^{2}=\mathbf{0 . 8 1}$ 
Height $\left(\mathrm{r}^{2}=0.11\right)$, body weight $\left(\mathrm{r}^{2}=0.03\right)$, ideal body weight $\left(\mathrm{r}^{2}=0.12\right)$, and BMI $\left(\mathrm{r}^{2}=\right.$ 0.00 ) did not correlate with the needle depth. The Bland-Altman analysis showed the bias of $1.8 \mathrm{~cm}$ and limits of agreement of $0.63 \mathrm{~cm}$ and $2.94 \mathrm{~cm}$ (Figure 3 ).

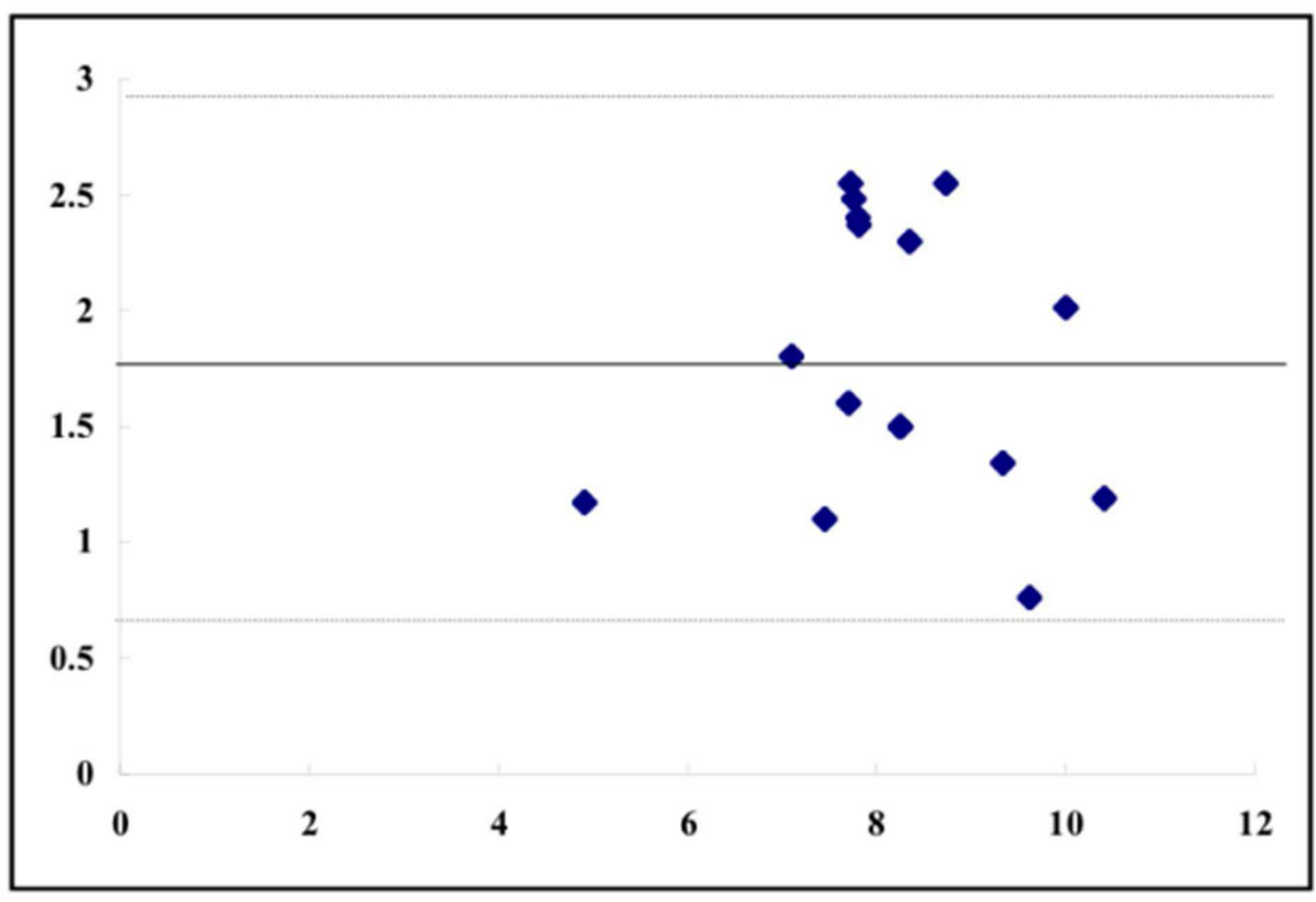

Figure 3: Bland Altman plot

The ultrasound depth was corrected by the angle of the needle to the skin. $\mathrm{Y}$ axix, cm, needle depth - corrected ultrasound depth; $\mathrm{X}$ axis, $\mathrm{cm},($ needle depth + corrected ultrasound depth)/2; solid line, biais; dotted lines, limits of agreement

\section{Discussion}

An epidural block is useful to decrease anesthetics and analgesics during surgery and to decrease postoperative pain; both have a great benefit on postoperative respiratory function in obese patients (von Ungern-Sternberg, et al, 2005). Postoperative epidural analgesia was associated with a decrease in left ventricular stroke work, systolic pressure-heart rate product, arteriovenoses oxygen content differences, and oxygen consumption, compared with values observed when patients experienced pain without epidural analgesia (Gelman, et al, 1980). Therefore, an epidural block should be used in bariatric surgery.
We successfully inserted an epidural catheter in all 16 bariatric patients using ultrasound. It was quite difficult to recognize spinous process manually; therefore, we used ultrasound to locate the spinous process and dura. Balki et al (2009) suggested that prepuncture ultrasound might be useful to facilitate the placement of epidural needles in obese patients.

The success rate of epidural puncture at the first attempt was $63.3 \%$ in obese parturients with BMI of $34.9 \mathrm{~kg} / \mathrm{m}^{2}$, and needle depth of $6.48 \mathrm{~cm}$ in the study by Qian et al (2012). Our success rate at the first attempt was $14 / 16$ (87.5 \%) with mean BMI of 45.5 $\mathrm{kg} / \mathrm{m}^{2}$ and needle depth of $9.1 \mathrm{~cm}$. The lower success rate of the study by Qian et al. might be because their patients were pregnant. 
Ultrasound depth may be $14 \%$ shorter to $17 \%$ longer than the needle depth in the study by Sahota et al (2013). However, Rasoulian et al. reported that the ultrasound depth tended to underestimate the needle depth as shown by the bias of $3.21 \mathrm{~mm}$, perhaps due to tissue compression by the probe or the intrinsic thickness of the ligamentum flavum (Rasoulian, et al, 2011). Tran et al. (2009) also reported that the bias between the needle depth and ultrasound depth $(4.8 \mathrm{~mm})$ could be attributed to the thickness of the ligamentum flavum (typically $5 \mathrm{~mm})$. The blunt Tuohy needle generates high pressure during passage through tissue (max. $180 \mathrm{mmHg}$ ), especially in the ligamentum flavum ( $>400 \mathrm{mmHg}$ ) (Grau, et al, 2001). This can cause a tissue deformation, which accounts for a difference between the needle depth and ultrasound depth. Our study showed that the corrected ultrasound depth was shorter about 0.8 to 2.5 $\mathrm{cm}$ than the needle depth, which was a greater difference than other studies (Grau, et al, 2001; Rasoulian, et al, 2011; Sahota, et al, 2013; Tran, et al, 2009). We measured the depth to the ligamentum flavum, which had 3 to $5 \mathrm{~mm}$ thickness, and we might compress the probe to get clearer view than other studies because we had more obese patients. These might be the reason of our greater difference between the needle depth and ultrasound depth compared to other studies (Grau, et al, 2001; Rasoulian, et al, 2011; Sahota, et al, 2013; Tran, et al, 2009).

Balki et al. (2009) showed that in obese parturients, ultrasound depth and needle depth of the lumbar epidural had a strong correlation with the Pearson correlation coefficient of 0.85 and concordance correlation coefficient of 0.79 . The needle depth was $6.6 \pm 1.0 \mathrm{~cm}$ and ultrasound depth was $6.3 \pm 0.8 \mathrm{~cm}$ in their study. Ultrasound depth is highly correlated to the needle depth observed during lumbar epidural placement (Tran, et al, 2009), while it is considered that the discussion is still necessary for thoracic epidural placement. Rasoulian et al. (2011) reported that during thoracic epidural catheterization, the limits of agreement are wide, which limits the predictive value of ultrasound-based measurements. Our study showed $\mathrm{r}^{2}$ value 0.81 and bias $1.8 \mathrm{~cm}$ with limits of agreements $0.63 \mathrm{~cm}$ and $2.94 \mathrm{~cm}$ when corrected ultrasound depth was used.
Therefore, the ultrasound depth could not be a surrogate measurement of the needle depth.

In conclusion, ultrasound was useful to insert a thoracic epidural catheter in obese patients receiving bariatric surgery, but ultrasound measurement of the depth to an epidural space could be used to know the needle insertion depth.

\section{Acknowledgements}

We used no fund in this clinical study and have no conflict of interest.

\section{References}

1. Adams, J.P., \& Murphy, P.G. (2000). “Obesity in anaesthesia and intensive care," British Journal of Anaesthesia, 85 (1) 91-108.

2. Balki, M., Lee, Y., Halpern, S., et al. (2009). "Ultrasound imaging of the lumbar spine in the transverse plane: The correlation between estimated and actual depth to the epidural space in obese parturients," Anesthesia \& Analgesia 108 (6) 1876-1881.

3. Gelman, S., Laws, H.L., Potzick, J., et al. (1980). "Thoracic epidural vs balanced anesthesia in morbid obesity: An intraoperative and postoperative hemodynamic study," Anesthesia \& Analgesia, 59 (12) 902-908.

4. Grau, T., Leipold, R.W., Conradi, R., et al. (2001). "Ultrasound control for presumed difficult epidural puncture," Acta Anaesthesiologica Scandinavica, 45 (6) 766771.

5. Nishiyama, T., Kohno, Y., \& Koishi, K. (2012). "Anesthesia for bariatric surgery," Obesity Surgery, 22 (2) 213-219.

6. Qian, W., Cheng, Y., \& Tian-long, W. (2012). "Ultrasound facilitates identification of combined spinal-epidural puncture in obese parturients," Chinese Medical Journal, 125 (21) 3840-3843.

7. Rasoulian, A., Lohser, J., Najafi, M., et al. (2011). "Utility of prepuncture ultrasound for localization of the thoracic epidural space," Canadian Journal of Anesthesia, 58 (9) 815823. 
8. Sahota, J.S., Carvalho, J.C.A., Balki, M., et al. (2013). "Ultrasound estimates for midline epidural punctures in the obese parturient: Paramedian sagittal oblique is comparable to transverse median plane," Anesthesia \& Analgesia, 116 (4) 829-835.

9. Tran, D., Kamani, A.A., Lessoway, V.A., et al. (2009). "Preinsertion paramedian ultrasound guidance for epidural anesthesia," Anesthesia \& Analgesia, 109 (2) 661-667

10.von Ungern-Sternberg, B.S., Regli, A., Reber, A., et al. (2005). "Effect of obesity and thoracic epidural analgesia on perioperative spirometry," British Journal of Anaesthesia, 94 (1) 121-127. 